\title{
Using Katalon for Mobile App Test Automation
}

\author{
Sahil Viday Singh ${ }^{1}$, Dr. Mahesh $\mathbf{A}^{2}$ \\ ${ }^{1}$ UG student, Department of Electronics and Communication Engineering \\ ${ }^{2}$ Associate Professor, Department of Electronics and Communication \\ RV College of Engineering, Bengaluru, India \\ 1Email: sahilsinghro7@gmail.com \\ 2Email: mahesha@rvce.edu.in
}

\begin{abstract}
Software testing is a study conducted to provide interested parties with information about the quality of the software product or service under test. Software testing also provides an objective independent perspective of the software. Helping companies recognize and understand the risks of software deployment. Testing technique includes the process of running a program or application with the aim of finding errors and verifying that the product is suitable for use. There are two ways of writing tests: manually and automatic. Certain ways of manual testing, which include exploratory testing and usable testing, are not that valuable. One can manually run other types of tests, such as regression tests and functional tests, anyways it doesn't really make sense for developers to repeat the exact process again and again. Because of this type of iterative test which results us to move towards testing automation.
\end{abstract}

Keywords: Katalon, Test Suites, API, Selenium, UFT, Testim, Xpath, TestOps, UI

\section{INTRODUCTION}

By Test automation we mean the process by which tests are made to run automatically, managing the data files related to tests, and using the results which will eventually be used to improve quality of the software. This is mainly done to achieve quality assurance, but the actions include the collaboration of the software team. From business analysts to developers, everyone needs to be involved to get the most out of test automation.

\section{Test Automation Process}

The very first thing which one needs to prepare the script is testing data file, and test execution environment. As all know, running almost all scripts needs the environment be already in some particular position before action can be taken. Under normal circumstances this requires manipulating data, putting the application into some particular state or also can be set to both. When the environment is already in some specific state, perform the action. Test controllers run tests by calling the software's API or UI, or by running the script as it is. The script pilot is the one which is responsible for "conducting" the test, but the test organizing method is responsible for coordinating everything, including: Report. These results are provided in a variety of formats and can even generate problem and error sheets in your job tracking system. However, the basic result is success or failure. Each test case usually has a green or red indicator indicating pass or fail. Test may not be final or may not run for some reason. This log will help you identify the problem. Ideally, once you have the solution in place, you can close the scenario. 


\section{Test Automation Tools}

Katalon Studio: Katalon Studio is something which can be used to design scripts that lows you to test not only web but as well as mobile applications and APIs. The solution uses the Selenium and Appium engines to provide users with an added environment that allows designers to work with various frameworks and methods.

Selenium: Selenium is already a well-known for test automation. This allows users to write scripts in various languages such as Java, C, Python, Perl, and Ruby. This tool can also be run on different operating systems and browsers. It is an extension which enables one to test basic recording and rerun the script. Next is the Selenium web controller. This is a type of API which enables one to interact with your browser using a scripting language. Third is the Selenium Grid. Here is a solution (also known as grid testing) for testing on different combinations of browsers and operating systems.

UFT: UFT is an early open source tool that allows testers to test desktop, web, and mobile applications. Currently, we also provide API testing functionality.

TestComplete: TestComplete also supports various types of testing which includes web, phone and desktop testing. C++ Script for scripting This tool has an element identification engine that can precisely identifies dynamic elements of the app under testing, especially useful for testing applications where the user interface use change frequently. Learn how to help developers create, run, and maintain test scripts. This tool also makes possible for developers to fastly design test scripts and execute them on multiple environments. The engine not only runs the script but also understands the data file on each run.

Testim: Testim then uses everything it learns to improve and make the test cases more stable. A robust test suite that won't break every time the code changes. Testim can detect changes in the properties of elements on web pages using an innovative feature called smart locators. In this way, if you change the properties of a particular element (that is, the id or CSS class), Testim will still be able to determine the position of the element and the test will not fail.

\section{Katalon for Automation}

Katalon Studio is a popular test automation tool developed by Katalon, Inc. According to the 2018 Smart-Bear Test Status Report, in 2018, the software achieved an interface market penetration of Katalon was certified Gartner Peer Insights Customer's Choice for Software Test Automation in March 2019 and March 2020.

1. In Katalon Studio there are two views available, one is for manual and other is for script. Then it is also possible for us to declare variable that can be global, public as well as private. 2. Katalon Studio allows user to create internal data as well as importing external data which can be excel, csv as well as from a database.

3. Test cases can be created and then it is also possible to create test suites by importing test cases. Then using data files for using external data is also an option which can be used to import data from an external source.

4. It is also possible to use command line interface for running the test scripts as well as test suites and get the reports after execution.

5. In version other from this, users can also use smart xpath as well as smart wait which are of great use. The latest version 8.0 provides native integration with Azure DevOps (ADO), allowing users to easily map Azure DevOps test cases to Katalon Studio's automated test case 


\section{Capturing Objects in Katalon}

For capturing the objects in Katalon one needs to connect the device and use the following way as shown in figure.

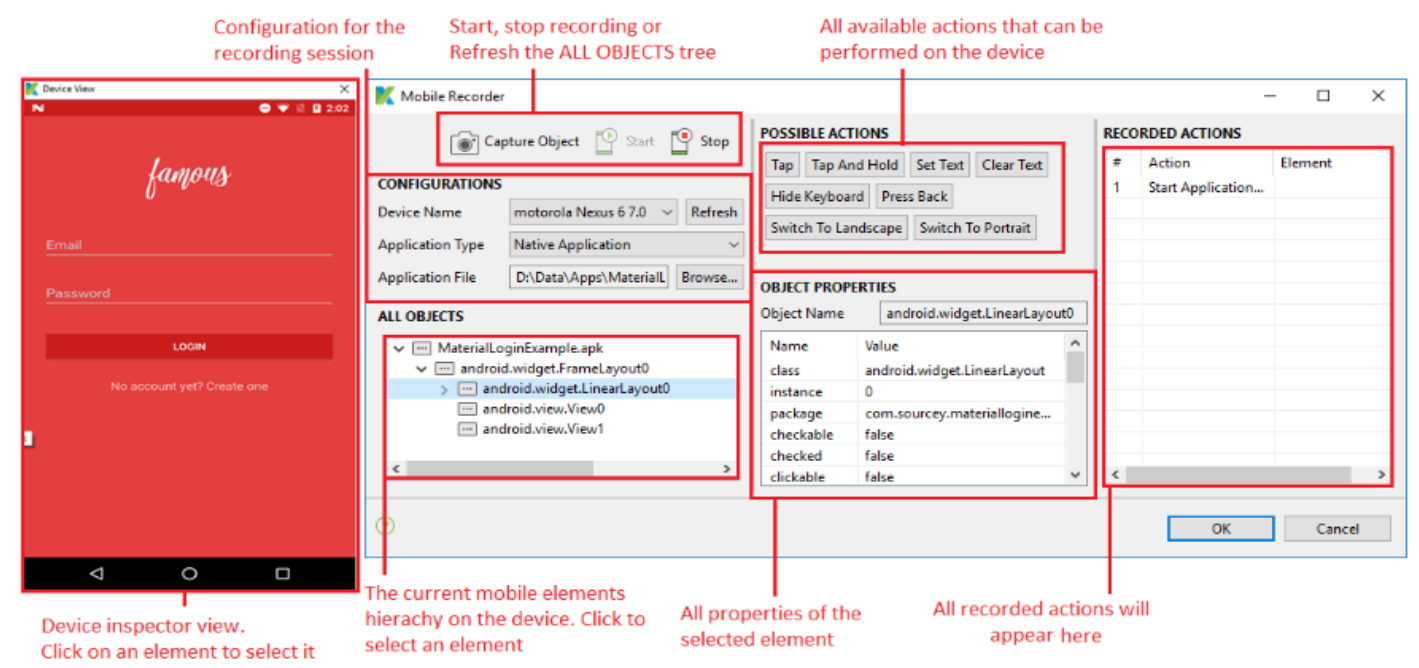

\section{Failure Handling}

The error handling parameters allow the user to decide whether to continue running Katalon Studio in the event of an error during execution. The various ways are 1. Stop in case of error- Katalon Studio will stop if an error occurs.

2. Continue on failure- Katalon Studio will continue regardless of runtime errors.

3. Optional Error step goes into warning state.

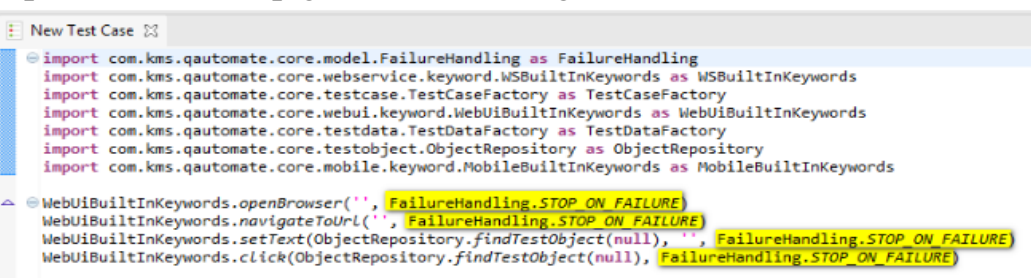

\section{Reports Generation}

After the execution of Test Suites, the reports are generated which can also be mailed to an email by providing the email in the Katalon TestOps integration 


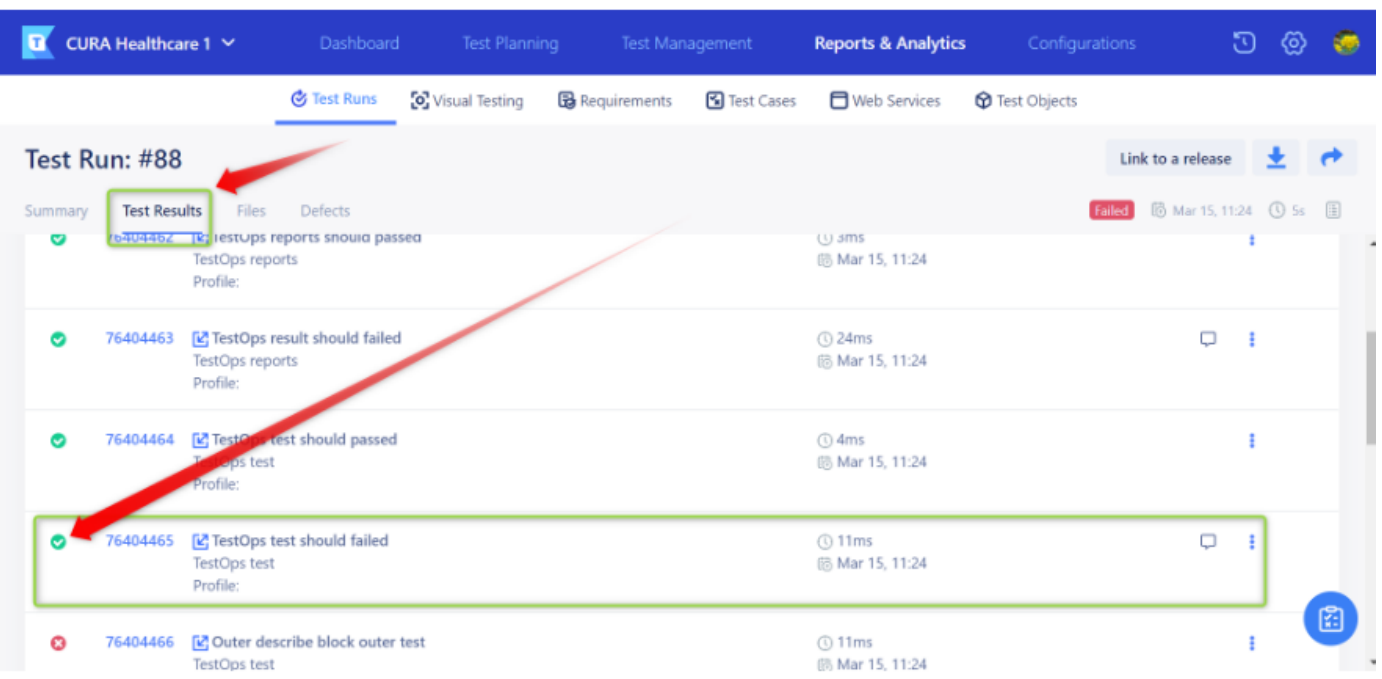

\section{Conclusion and Future Scope}

With rapid advancements in technology, the need to complete projects faster has never been greater. To complete the project faster, the entire steps taken in the software life cycle must also be accelerated made to save time and money, but only for use on time-consuming projects. For regression testing and large-scale test execution, auto mated testing is the best solution. It is a good choice. Test automation includes improved software quality, reduced manual software testing work, elimination of redundant test work, repetitive software test creation, systematic iteration, reduced repetitive work, and improved test result consistency. Run more software tests in a limited time to improve test coverage. Productivity, etc. This is an efficient way to reduce manual work during regression, as well as to run functional test cases. If the script is highly developed, no error will occur, which will be greatly reduced. Now, in the coming days, automation will be considered more economical in terms of quality and time

\section{REFERENCES}

[1] J.-W. Lin and S. Malek, "Test Automation in Open-Source Android Apps: A Large Scale Empirical Study", IEEE International Conference on Software Engineering and Testing, Spain,2020.

[2] M. Linares-V'asquez and D. Poshyvanyk, "Repair of Mobile Test Scripts", IEEE International Conference on Software Maintenance and Evolution, Italy,2019.

[3] F. Behrang and A. Orso, "Test Migration between Mobile Apps with Similar Functionality", 34th IEEE/ACM International Conference on Automated Software Engineering (ASE),Bologna,2019

[4] R. Coppola and M. Morisio, "Scripted GUI testing of Android open-source apps: evolution of test code and fragility causes", IEEE international symposium on empirical software engineering and measurement, Nice, 2017.

[5] H. Tan and Z. Dong, Repairing Crashes in Android Apps. In 2018 IEEE/ACM 40th International Conference on Software Engineering (ICSE),Lyon,2019 
[6] G. Fraser and A. Arcuri, "Test Transfer across Mobile Apps through Semantic Mapping”, IEEE International Conference on Software Engineering, Southampton, 2017.

[7] J. Lin and R. J. 2019, "Test Transfer across Mobile Apps through Semantic Mapping”,34th IEEE/ACM International Conference on Automated Software Engineering (ASE), Italy, 2019.

[8] W. Choi and K. Sen, "Minimizing Android GUI Test Suites for Regression Testing”, IEEE/ACM 40th International Conference on Software Engineering (ICSE), Italy,2019. [9] Katalon Documentations," Welcome to Katalon Docs | Katalon Docs".

[10] A. D. Lucia and F. Palomba, "Testing of Mobile Applications in the Wild: A Large Scale Empirical Study on Android Apps", 28th IEEE/ACM International Conference on Program Comprehension (ICPC), Leeds,2020 